Casos Clínicos

Arch. Esp. Urol., 59, 6 (627-631), 2006

\section{PRESENCIA DE MALLA DE PROLENE EN VAGINA.}

Anna Bujons, Carlos Errando, Manel Prados', Carlos Báez, Pedro Arañó y Humberto Villavicencio.

Unidad de Urología Funcional y Femenina. Servicio de Urología Fundació Puigvert. Barcelona.

Adjunto' de Urología Hospital Juan XXIII Tarragona. España.

Resumen.- OBJETIVO: Describir un caso clínico de una paciente en la que tras la colocación de malla de Prolene $\AA$ como refuerzo en la corrección de cistocele recidivado, presenta complicaciones.

MÉTODO/RESULTADO: Paciente mujer de 75 años de edad con antecedentes de corrección de cistocele con malla de prolene presenta incontinencia de orina y erosión vaginal por la propia malla.

CONCLUSIONES: El uso de malla para la corrección de cistocele tiene la ventaja de aportar mayor soporte a la colporrafia y evitar la recidiva del cistocele, aunque puede presentar complicaciones como en nuestro caso clínico de migración de la malla, aparición de fístula, infección o intolerancia.
Palabras clave: Malla de prolene. Incontinencia de orina. Cistocele.

Summary.- OBJECTIVE: To describe the clinical case of a patient presenting complications after the implantation of a Prolene mesh as reinforcement for the correction of a recurrent cystocele.

METHODS /RESULTS: Seventy-five-years-old female patient with history of previous cystocele repair with Prolene mesh presenting urinary incontinence and vaginal erosion of the mesh.

CONCLUSIONS: The use of mesh for the repair of cystoceles has the advantage of providing greater support to the colporrhaphy and avoiding recurrences. Nevertheless, it may present complications as in our reported case with mesh migration, fistula, infection, or intolerance.

Keywords: Prolene mesh. Urinary incontinence. Cystocele.

\section{CASO CLÍNICO}

Paciente mujer de 75 años de edad, con antecedentes patológicos epilepsia y como antecedentes quirúrgicos: Histerectomía abdominal más plastias anterior y posterior en 1992 por prolapso vaginal, sigmoidectomía y anastomosis termino-terminal + plicatura rectal y fijación a sacro + colporrafia anterior con malla en 2002 por descenso de suelo pélvico y rectocele.

Refiere tras la cirugía incontinencia urinaria inmediata a moderados esfuerzos (I.U.E.) (toser, reír y caminar) y ocasionalmente incontinencia inadvertida. Chorro con fuerza, con sensación de vaciado incompleto y prensa abdominal en ocasiones.

No presenta síntomas de llenado.

En la Exploración Física:

- Uretra fija. Q. tip test: negativo.

- Escape franco con la tos.

- No rectocele ni cistocele.

- Palpación de irregularidad a nivel cara anterior vaginal en el margen derecho.

- Ausencia de celes.

Como exploraciones complementarias: vaginoscopia observando fragmento de malla calcificada extruida a 
nivel vaginal. Cistoscopia: ausencia de cuerpos extraños endovesicales. EUD con los resultados siguientes:

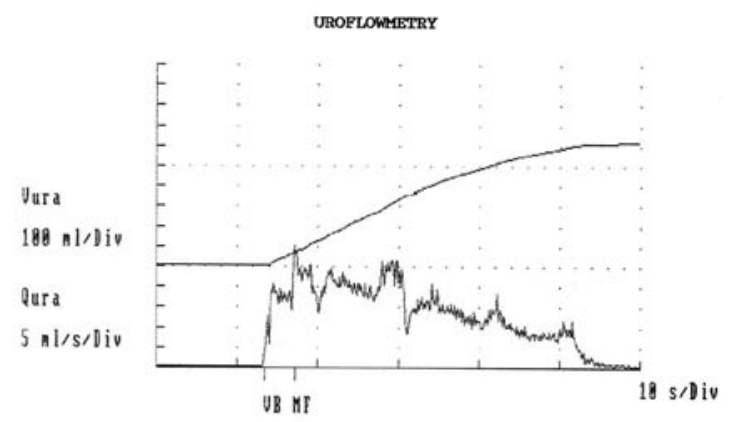

RESULTS
MaX FION

Max Flow Rate

Volded Volume

Flou Time

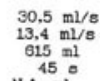

$615 \mathrm{ml}$
N.A. $\mathrm{ml}$

POST

Flujometría fisiológica: Normal.

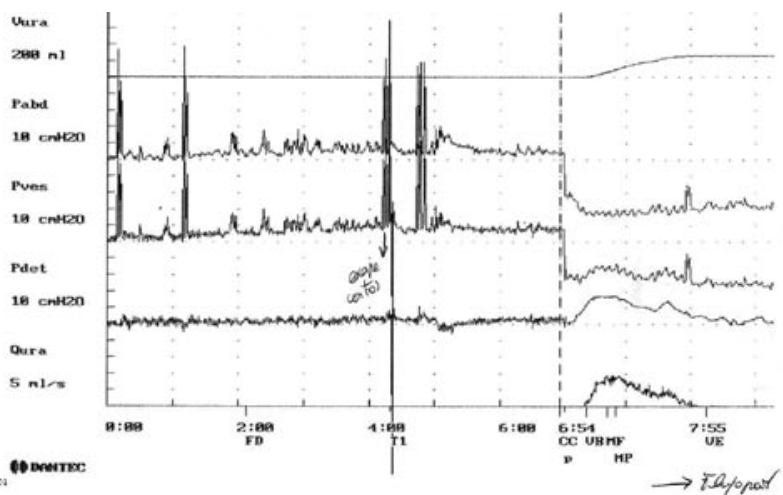

Cistomanometría y Presión-Flujo: Comportamiento sensitivo-motor normal durante el estudio. Se observa escape con la tos. Fase de vaciado normal.

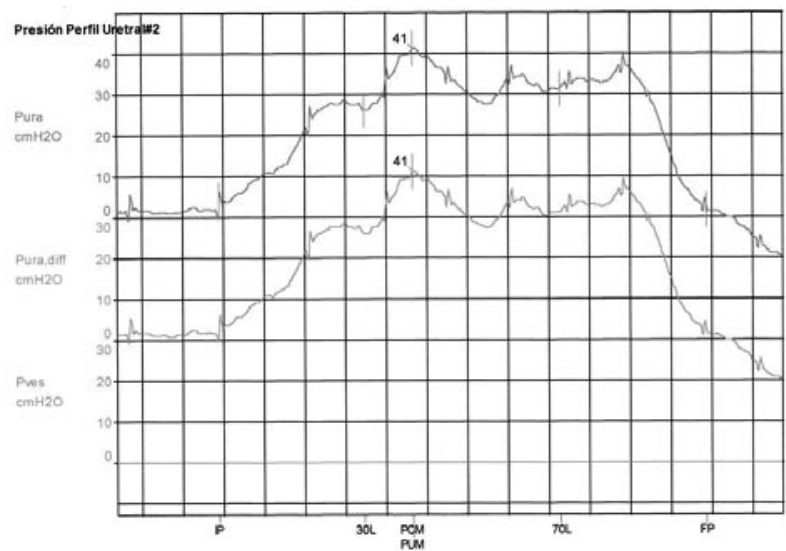

Perfil uretral: Normal
Dado que se trata de una paciente con I.U.E. tipo III con uretra fija en la exploración, se decide implantar un cabestrillo de tensión regulable tipo Remeex y exéresis parcial de la malla extrusionada a nivel cara anterior vaginal.

En las Figuras 1, 2 y 3 se visualiza la malla en vagina durante el acto quirúrgico con extracción de la misma y en la Figura 4, la malla ya extraída.

Curso postoperatorio sin incidencias. Previo al alta se realiza prueba de Pad test y prueba de la tos sin evidenciar escape.

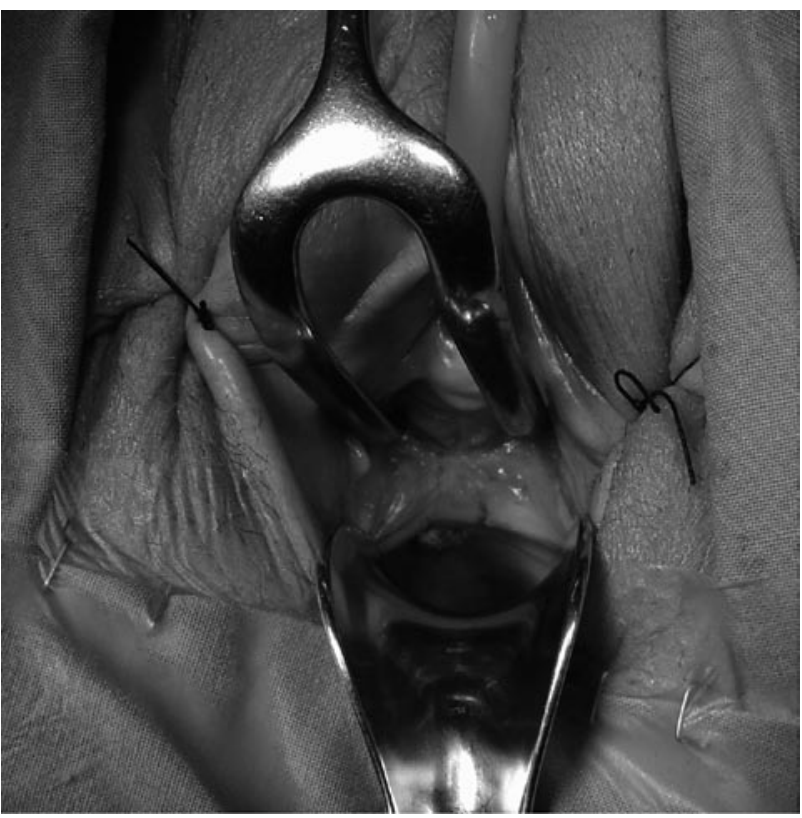

FIGURA 1.

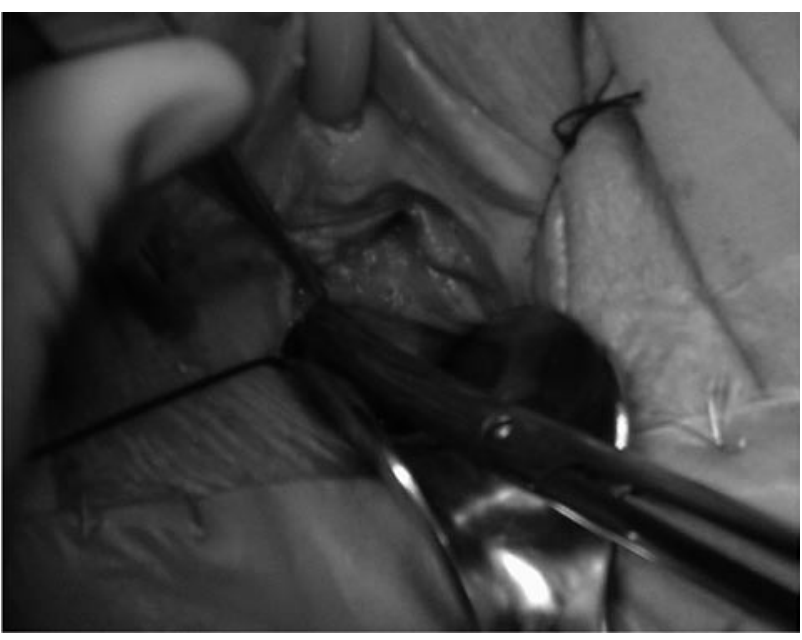

FIGURA 2. 


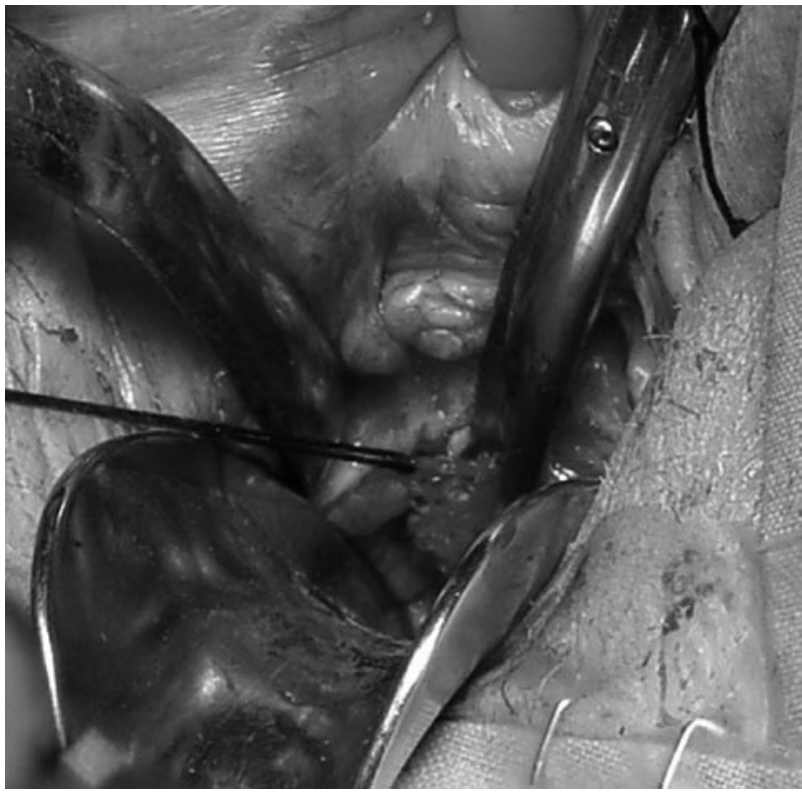

FIGURA 3.

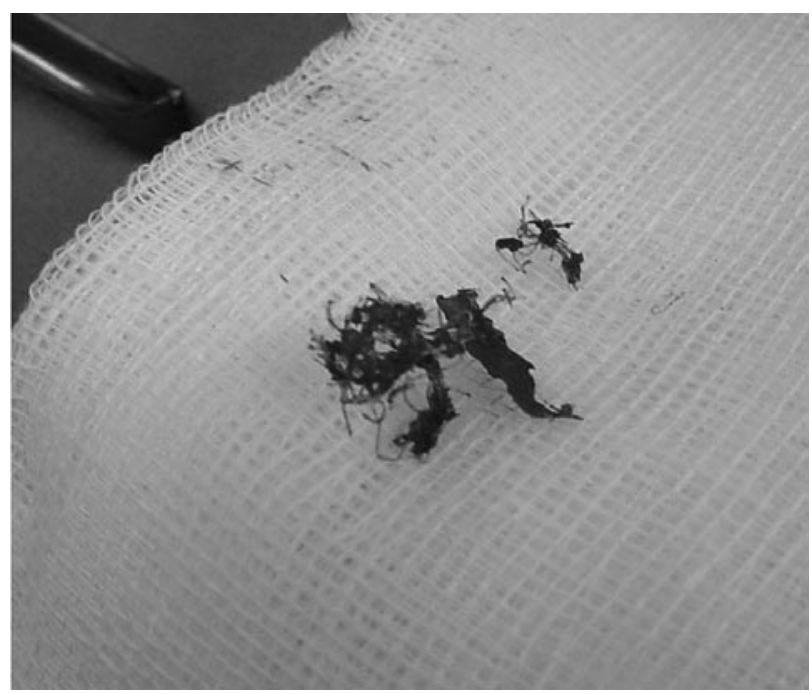

FIGURA 4.

\section{DISCUSIÓN}

El cistocele es la prominencia más o menos destacada de la vejiga por vagina, cuya causa reside en la alteración de los medios de fijación de la porción anterior de la pelvis que son la fascia umbilicoprevesical, ligamento umbilical medio y ligamentos pubovesicales.

Los mecanismos de sostén vaginal son:

- Fascia endopélvica.

- Músculo elevador del ano.

- Esfínter valvular de la propia vagina.
Podemos dividir el compartimento anterior en zonas con predominio de una estructura anatómica que aporta mayor soporte; el déficit de sostén de la misma ocasiona la aparición de cistocele. Los fallos del compartimento anterior pueden dividirse en:

- Defecto central - Más frecuente. Fallo de la aponeurosis pubocervical.

- Defecto lateral o paravaginal - Alteración del arco tendinoso de la aponeurosis endopelviana.

- Defectos transversos - De menor interés clínico. Tratamiento igual que el defecto central.

El diagnóstico es, ante todo, clínico. La exploración clínica tiene mucha importancia porque permite enumerar los trastornos asociados al cistocele, hecho imprescindible para cualquier decisión terapéutica.

En el trascurso del cistocele, el cierre del ángulo vesicouretral crea una seudoválvula del orificio vesical que mantiene una falsa continencia. Se explica así el carácter providencial de algunos prolapsos para la incontinencia urinaria.

Se han elaborado varias clasificaciones clínicas de los cistoceles con objeto de normalizar la intervención quirúrgica.

\section{- Clasificación ordinal del prolapso:}

Se definen distintos estadíos según la porción más prominente.

Esquemáticamente esta clasificación puede definirse así:

- Estadío 0: todo se encuentra en su sitio.

- Estadío 1: todo se encuentra dentro de la vagina.

- Estadío 2: el punto más bajo se encuentra en la vulva.

- Estadío 3: el prolapso se exterioriza.

- Estadío 4: el prolapso está totalmente exteriorizado,

- Clasificación de Baden y Walter por su facilidad de comprensión y rapidez de ejecución. La vagina queda separada en tres segmentos (anterior, superior y posterior) que a su vez se dividen en dos compartimentos cada uno: uretra y vejiga en el segmento anterior, útero y fondo de saco de Douglas en el superior, y recto y peritoneo en el posterior. Estos seis compartimentos se puntúan de 0 a 4 tomando referencia el himen durante las maniobras de esfuerzo abdominal. Esta clasificación es descriptiva y no cualitativa.

- La clasificación de la ICS pretendía ser objetiva y reproducible describiendo las condiciones de examen y de los estudios accesorios para obtener así una idea cuantitativa y una clasificación ordinal del prolapso.

- Las condiciones de examen: Recomienda describir los segmentos vaginales prolapsados sin recurrir al esfuerzo abdominal máximo. 
- Descripción cuantitativa del prolapso: Estas medidas se toman en relación con puntos anatómicos de referencia:

- El himen es el punto 0; todo lo que queda dentro es negativo y lo que sale positivo.

- Un punto accesorio, el plano de la espina ciática (donde suele hallarse el fondo de vagina).

Por lo tanto POPQ (Pelvic Organ Prolapse Quantification ICS- AUS) describe segmentos vaginales prolapsados sin presunción de su contenido, ni de maniobras de Valsalva.

- La descripción cuantitativa del prolapso, puntos anatómicos osegmentos.

Seis puntos anatómicos que se definen de la siguiente manera: (Figura 5)
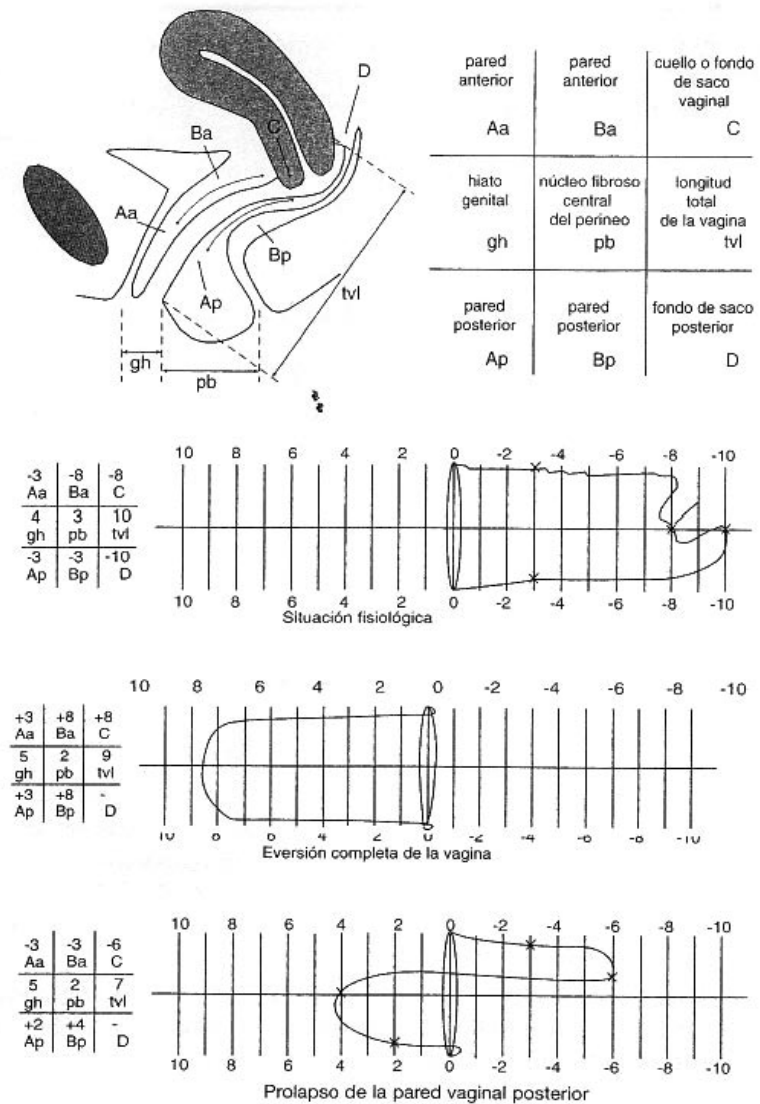

FIGURA 5. Descripción cuantitativa del prolapso, puntos anatómicos, segmentos.
- Pared anterior vaginal:

- Aa, situado $3 \mathrm{~cm}$ por dentro del meato (unión uretrovesical), varía de $-3 a+3 c m$.

- Ba, segmento vaginal más bajo, situado entre Aa y el fondo de saco anterior (tercio proximal de la vagina). Si no existe cistocele anterior (tercio proximal de la vagina). Si no existe cistocele anterior, ba y Aa se confunden $(-3 \mathrm{~cm})$;

- Segmento medio de la vagina:

- Referencia del cuello uterino o fondo de saco de la vagina después de la histerectomía total;

- $D$ es el fondo de saco posterior.

- Pared vaginal posterior:

-Ap, $3 \mathrm{~cm}$ por dentro del himen.

$-B p$, por analogía con la pared anterior. El cistocele posterior incluye el rectocele, el elitrocele, o ambas, en esta fase de la clasificación.

$\Rightarrow$ Tres segmentos:

- Hiato genital $(\mathrm{Hg})$ : distancia uretrohimeneal.

- Longitud perineal (lp): distancia anohimeneal.

- Longitud vaginal total (lvt): profundidad máxima de la vagina medida con una bugía vaginal graduada.

Las mediciones se deben tomar en centrímetros en una regla.

La clasificación de la ICS, es, sin duda, objetiva, precisa (cuantitativa) y rigurosa. Sin embargo resulta poco reproducible, lo que limita necesariamente su uso.

Para el tratamiento global del cistocele es necesario tener en cuenta no sólo el cistocele y los trastornos pélvicos asociados sino también la edad d ela paciente y sus deseos de procreación futura y conservación de una vida sexual activa. Al conocer mejor alas alteraciones de la estática pélvica se ha puesto énfasis en las medidas profilácticas y en la utilidad del tratamiento médico y de tratamiento de la reposición hormonal.

El cistocele sin ninguna alteración de la estática pélvica, se trata de forma aislada mediante diferentes técnicas:

- RETROPÚBICA

- LAPAROSCÓPICA

- VAGINAL (la más utilizada)

- Colporrafia anterior

- Colporrafia anterior más punto de Kelly

- Colporrafia anterior más malla

La función principal de las mallas en la corrección del cistocele es aportar mayor soporte. Hay diversos estudios en la literatura sobre los diferentes materiales de las mallas, tolerancia y eficacia de las mismas. 
P. Debonance (4) realiza una clasificación del tipo de material de las mallas para cirugía ginecológica:

- I. Mallas macroporosas (Poros $>75 \mu$ ) Atrium, Marlex, Prolene, Trelex. Tamaño necesario para colonizarse por macrofagos, fibroblastos.

- II. Mallas microporosas Gore-Tex. (Poros < 10 $\mu$ ).

- III. Malla macroporosa con multifilamentos PTFE (Teflon), PTFE perforado (Mycromesh).

- IV. Biomaterial con poros submicrométricos.

J. Thomas describe la eficacia y complicaciones del uso de malla de Marlex en la reparación del cistocele recurrente realizando un estudio con 24 pacientes con 2 o más cirugías correctoras de cistocele: 12 colporrafias anteriores con malla y 12 sin malla. Se presentaron $4(25 \%)$ recurrencias en pacientes sin malla y ninguna en los pacientes con malla, pero 3 pacientes con malla $(20 \%)$ presentaron erosión vaginal con posterior exéresis de la malla.

R. Migliari et al.analiza la eficacia de la malla de polipropileno en la corrección del cistocele grado III en un estudio de 12 pacientes con edad media: 65.6 años, 5 asociaban a IOE (4 tipo II y 1 tipo III), todas cirugías previas $7 / 12$ histerectomia +/- Burch +/- colporrafia ant, $2 / 12$ colporrafia anterior y $3 / 12$ suspensión del cuello. Tras un periodo de seguimiento medio 20.5 meses (1532) 9 casos $(75 \%)$ curados, $3(25 \%)$ recidiva del prolapso grado I, 1 enterocele y 2 urgencias de novo, sin complicaciones mayores.

Las principales complicaciones en el uso de la malla son intolerancia, infección, fístula, erosión de malla entre 2$7 \%$.

\section{CONCLUSIÓN}

El uso de malla para la corrección de cistocele tiene la ventaja de aportar mayor soporte a la colporrafia y evitar la recidiva del cistocele, aunque puede presentar complicaciones como en nuestro caso clínico de migración de la malla, aparición de fístula, infección o intolerancia.

Las características que debería tener la malla ideal serían:

- No debe ser modificada físicamente por los fluidos tisulares

- Inerte químicamente

- No cancerígena

- No debe provocar reacción inflamatoria o de antíge- no-anticuerpo

- Debe resistir una tensión mecánica

- Posibilidad de fabricarse

- Debe poder esterilizarse

- Resistencia a la infección

- Barrera de adherencia sobre superficie de contacto de las visceras

- Mejor respuesta in vivo que un tejido autólogo

\section{BIBLIOGRAFÍA y LECTURAS RECOMENDADAS (*lectura de interés $y^{* *}$ lectura fundamental)}

**1. MAUROY, J.C.; FANTONI, J.C.; LAPRAY.: "Encyclopedie Médico-Chirurgicale".

2. JULIAN, THOMAS, M.M.D.: "The efficacy of Marlex in the repair of severe, recurrent vaginal prolapse of the midvaginal wall". Obstet.Gynecol; 175: 1472. 1996.

3. MIGLIARI, R.; DE ANGELIS, M.; MADEBBU, G.: "Tension-free vaginal mesh repair for anterior vaginal wall prolapse". Eur Uro; 38:151. 2000

**4. DÉBONANCE, P. et al.: Évolution des matériaux prothétiques vers une meilleure tolérance : application en chirurgie gynécologique. J Gynecol Obstet Biol Reprod; 31 : 527. 2002.

5. MIGLIARI, R.; DE ANGELIS, M.: "Tension-Free Vaginal Mesh repair for anteior vaginal wall prolapse". Eur Urol, 38; 151. 2000. 